\title{
Human embryonic stem cells can differentiate into myocytes with structural and functional properties of cardiomyocytes
}

\author{
Izhak Kehat, ${ }^{1}$ Dorit Kenyagin-Karsenti, ${ }^{2}$ Mirit Snir, ${ }^{1}$ Hana Segev, ${ }^{2}$ Michal Amit, ${ }^{2}$ \\ Amira Gepstein, ${ }^{1,3}$ Erella Livne, ${ }^{3}$ Ofer Binah, ${ }^{4}$ Joseph Itskovitz-Eldor, ${ }^{2}$ and Lior Gepstein ${ }^{1}$ \\ ${ }^{1}$ Cardiovascular Research Laboratory, Department of Physiology and Biophysics, \\ ${ }^{2}$ Department of Obstetrics and Gynecology, Rambam Medical Center, \\ ${ }^{3}$ Department of Anatomy, and \\ ${ }^{4}$ Bernard Katz Center for Cell Biophysics, The Bruce Rappaport Faculty of Medicine, Technion-Israel Institute of Technology, \\ Haifa, Israel
}

Address correspondence to: Lior Gepstein, Cardiovascular Research Laboratory, The Bruce Rappaport Faculty of Medicine, Technion-Israel Institute of Technology, 2 Efron Street, PO Box 9649, 31096 Haifa, Israel.

Phone: 972-4-829-5303; Fax: 972-4-829-5333; E-mail: mdlior@tx.technion.ac.il.

Or to: Joseph Itskovitz-Eldor, Department of Obstetrics and Gynecology, Rambam Medical Center and

the Bruce Rappaport Faculty of Medicine, Technion, Haifa, Israel.

Phone: 972-4-8542536; Fax: 972-4-8542503; E-mail: Itskovitz@rambam.health.gov.il.

Izhak Kehat and Dorit Kenyagin-Karsenti contributed equally to this work.

Received for publication January 5, 2001, and accepted in revised form May 31, 2001.

The study of human cardiac tissue development is hampered by the lack of a suitable in vitro model. We describe the phenotypic properties of cardiomyocytes derived from human embryonic stem (ES) cells. Human ES cells were cultivated in suspension and plated to form aggregates termed embryoid bodies (EBs). Spontaneously contracting areas appeared in $8.1 \%$ of the EBs. Cells from the spontaneously contracting areas within EBs were stained positively with anti-cardiac myosin heavy chain, anti- $\alpha$-actinin, anti-desmin, anti-cardiac troponin I (anti-cTnI), and anti-ANP antibodies. Electron microscopy revealed varying degrees of myofibrillar organization, consistent with early-stage cardiomyocytes. RT-PCR studies demonstrated the expression of several cardiac-specific genes and transcription factors. Extracellular electrograms were characterized by a sharp component lasting $30 \pm 25$ milliseconds, followed by a slow component of $347 \pm 120$ milliseconds. Intracellular $\mathrm{Ca}^{2+}$ transients displayed a sharp rise lasting $130 \pm 27$ milliseconds and a relaxation component lasting 200-300 milliseconds. Positive and negative chronotropic effects were induced by application of isoproterenol and carbamylcholine, respectively. In conclusion, the human ES cell-derived cardiomyocytes displayed structural and functional properties of early-stage cardiomyocytes. Establishment of this unique differentiation system may have significant impact on the study of early human cardiac differentiation, functional genomics, pharmacological testing, cell therapy, and tissue engineering.

J. Clin. Invest. 108:407-414 (2001). DOI:10.1172/JCI200112131.

\section{Introduction}

The study of early human cardiomyocyte development is hampered by the lack of a suitable model. Hence, valuable information regarding the differentiation of early human cardiac precursor cells, the development of excitability, excitation-contraction coupling, and the molecular signals involved in these processes is largely lacking. In addition, adult cardiomyocytes permanently withdraw from the cell cycle and therefore cannot regenerate. Hence, significant loss of cardiomyocytes is irreversible and leads to the development of progressive heart failure. A potential novel therapeutic approach for this situation is to increase the number of functional myocytes within the depressed region by implantation of myogenic cells. Recent studies demonstrated that fetal cardiomyocytes could survive in infarcted tissue, differentiate, and possibly improve cardiac performance $(1,2)$. Given that human fetal tissue cannot be obtained in sufficient quantities in the clinical setting, a new source of human cardiomyocytes is sorely needed.

Embryonic stem (ES) cells are continuously growing stem cell lines of embryonic origin, first isolated from the inner cell mass of mouse blastocysts (3). These unique cells are characterized by their capacity to proliferate in an undifferentiated state for a prolonged period in culture, and by their ability to differentiate into every tissue type in the body. The advent of murine ES cells has provided important insights into the early steps of development of excitability in the mammalian heart, including patterns of gene expression, myofibrillogenesis, ion channel development and function, receptor development, and calcium handling (4-9). 


\section{Figure 1}

Stages in EB production and differentiation. (a) Schematic of the three stages in human ES cell differentiation. Initially, the ES colonies are grown on top of the MEF feeder layer (left). To induce differentiation, cells are transferred to suspension, where they aggregate to form EBs (middle). After 10 days in suspension, EBs are plated on gelatin-coated culture dishes, where they are observed for the appearance of spontaneous contractions (right). (b) Photomicrographs depicting the just-mentioned three stages: ES colony (left), EBs in suspension (middle), and a contracting area in the outgrowth on an EB (right, arrow).
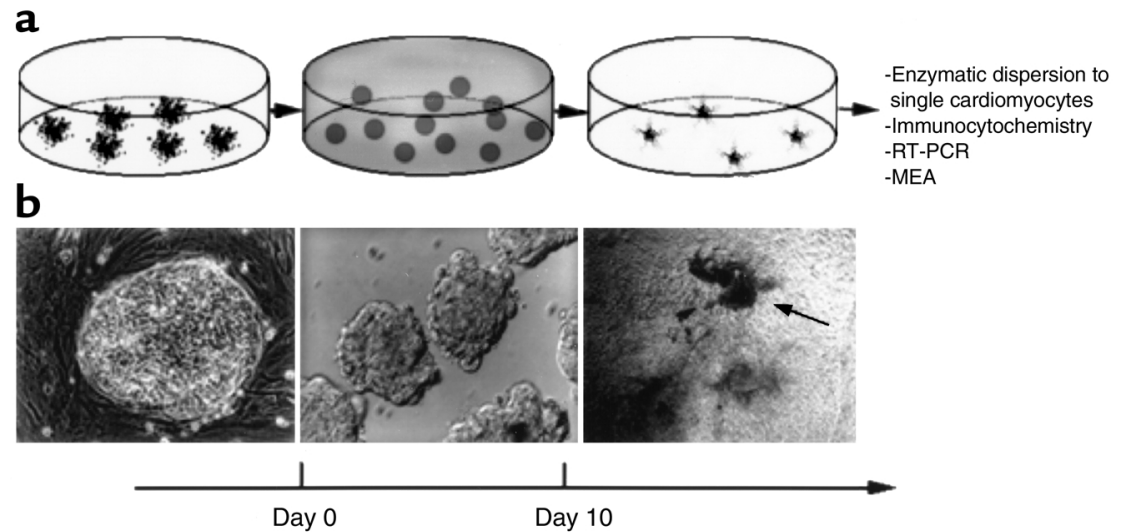

Given the outstanding potential demonstrated by mouse ES cells, it is not surprising that much effort has been spent on the development of human ES cell lines. This quest ended recently when two groups described the generation of human blastocyst-derived ES cell lines $(10,11)$. The human ES cells were demonstrated to fulfill all the criteria defining ES cells, namely, derivation from the pre- or peri-implantation embryo, prolonged undifferentiated proliferation under special conditions, and the capacity to form derivatives of all three germ layers. Hence, when cultured with mitotically inactivated mouse embryonic fibroblast (MEF) feeder layer, they could be maintained in the undifferentiated state for prolonged periods. Further studies revealed that when human ES cells were allowed to spontaneously differentiate, they formed embryoid bodies (EBs) containing derivatives of all three germ layers (12). More recently, the effects of different growth factors on differentiation into various lineages were tested in this system (13).

In this report, we describe the use of human ES cells as a reproducible differentiation system for human cardiomyocytes. Using this system, spontaneously contracting foci were, for the first time to our knowledge, demonstrated to have ultrastructural and functional properties consistent with a cardiomyocytic phenotype.

\section{Methods}

ES cell preparation and production of EBs. Human undifferentiated ES cells of the single-cell clone H9.2 (14) were grown on mitotically inactivated (mitomycin C) MEF feeder layer in culture medium as described previously (10). The culture medium consisted of $80 \%$ knockout DMEM (no-pyruvate, high-glucose formulation; Life Technologies Inc., Rockville, Maryland, USA) supplemented with $20 \%$ FBS (HyClone, Logan, Utah, USA), $1 \mathrm{mM}$ L-glutamine, $0.1 \mathrm{mM}$ mercaptoethanol, and $1 \%$ nonessential amino acid stock (all from Life Technologies Inc.).

To induce differentiation, ES cells were dispersed to small clumps (three to 20 cells) using collagenase IV (Life Technologies Inc.; $1 \mathrm{mg} / \mathrm{ml}$ for 20 minutes). The cells were then transferred to plastic Petri dishes (Miniplast, Ein Shemer, Israel), at a cell density of about $5 \times 10^{6}$ cells in a $58-\mathrm{mm}$ dish, where they were cultured in suspension for 7-10 days. During this stage, the cells aggregated to form EBs, which were then plated on $0.1 \%$ gelatin-coated culture dishes, at a density of one to five EBs in a $1.91-\mathrm{cm}^{2}$ well, and observed microscopically for the appearance of spontaneous contractions. Figure 1a is a schematic summarizing the different stages in EB generation.

To assess the efficacy of the cardiomyocytic differentiating system, 1,884 EBs were plated on gelatin-coated dishes and monitored microscopically daily for the presence of contractions for up to 30 days after plating. The percentage of EBs displaying contracting areas, as well as the distribution of the timing of onset of spontaneous beating, was evaluated. In a preliminary study, we noted that varying ES cell density input in the suspension phase, although modifying the number of EBs produced, did not affect the percentage of beating EBs. Similarly, screening of two different lots of serum also did not modify the cardiomyocytic yield significantly. In addition, the effect of DMSO, a known stimulant, on differentiation into cardiomyocytic lineage was assessed by adding DMSO (Sigma Chemical Co., St. Louis, Missouri, USA) at a concentration of $0.75 \%$ ( $\mathrm{vol} / \mathrm{vol}$ ) to the culture medium during the 10 days of growth in suspension. The percentage of contracting EBs and the timing of onset of spontaneous contractions were examined microscopically in 454 EBs.

Immunostaining. Contracting areas were mechanically dissected using a pulled-glass micropipette. These areas were then enzymatically dispersed using trypsinEDTA (0.5\% trypsin, 0.53 mM EDTA; Life Technologies Inc.) for 15 minutes at $37^{\circ} \mathrm{C}$. Cells were plated on laminin-coated glass coverslips at a low density to allow visualization of individual cells, incubated for 48 hours, fixed using $4 \%$ paraformaldehyde with sucrose, and permeated using $0.5 \%$ Triton X-100 (Sigma Chemical Co.). Cells were blocked with $10 \%$ BSA and incubated with primary antibodies overnight at $4^{\circ} \mathrm{C}$. Primary antibodies used were mAb's for myosin cardiac heavy chain $\alpha / \beta$ at a dilution of 1:50, mAb's for cardiac muscle troponin I at a dilution of 1:5,000, mAb's for desmin at a dilution of 1:100, and polyclonal antibodies for atrial natriuretic peptide (ANP) at a dilution of 
1:250 (all from Chemicon International Inc., Temecula, California, USA). Staining of sarcomeric $\alpha$-actinin and nebulin was performed using anti-sarcomeric $\alpha$-actinin mAb's at a dilution of 1:800 and anti-nebulin mAb's at a dilution of 1:200 (both from Sigma Chemical Co.), respectively. After three washes with PBS, cells were incubated with secondary donkey antimouse, FITC-conjugated IgG antibodies, absorbed against human tissue for myosin heavy chain, desmin, and troponin I staining, or rhodamine-conjugated anti-rabbit IgG antibodies for $\alpha$-actinin, ANP, and nebulin staining (both from Chemicon International) at a dilution of 1:100 for 1 hour at room temperature. Preparations were examined using fluorescence microscopy. Dispersed cells isolated from noncontracting EBs served as controls.

RT-PCR. Total RNA from undifferentiated ES cells and contracting EBs was extracted using TRI reagent kit (Sigma Chemical Co.) according to the manufacturer's instructions. cDNA was synthesized from $1 \mu \mathrm{g}$ total RNA using SuperScript II reverse transcriptase (Life Technologies Inc.). cDNA samples were subjected to PCR amplification with primers selective for human cardiac genes. The PCR primers and the reaction conditions used are described in Table 1. The PCR products were size fractionated by $2 \%$ agarose gel electrophoresis.

Electron microscopy. For transmission electron microscopy, the spontaneously contracting areas were mechanically dissected. The tissues were fixed in $3 \%$ glutaraldehyde in $0.1 \mathrm{M}$ cacodylate buffer $(\mathrm{pH} 7.4)$ at $4^{\circ} \mathrm{C}$ for 24 hours, postfixed in $1 \% \mathrm{OsO}_{4}$ in the same buffer for 1 hour, dehydrated in graded ethanols, and embedded in Epon 812. Thin $(60-90 \mathrm{~nm})$ sections were used for ultrastructural evaluation using a JEOL USA Inc. 100 SX transmission electron microscope (Peabody, Massachusetts, USA) operating at $80 \mathrm{kV}$.
Extracellular electrophysiological recording and pharmacological studies. Intact contracting areas within the EBs were mechanically dissected using a pulled-glass micropipette and plated on gelatin-coated multielectrode arrays (MEA; Multi Channel Systems MCS $\mathrm{GmbH}$, Reutlingen, Germany) (18). The MEA consists of 60 titanium nitride electrodes with gold contacts 30 $\mu \mathrm{m}$ in diameter with an interelectrode distance of 200 $\mu \mathrm{m}$. The contracting areas were plated on top of the MEAs, and cells were confluent over the electrodes. However, in some EBs with relatively small contracting areas, the contracting area did not cover all electrodes; hence, recordings were performed from fewer than 60 electrodes. Extracellular signals were recorded simultaneously from all 60 electrodes at $25 \mathrm{kHz}$ and were band-pass filtered from 1 to $3,000 \mathrm{~Hz}$. Recordings were performed in culture medium at $37^{\circ} \mathrm{C}$. A pH of 7.4 was maintained using perfusion with air containing $5 \% \mathrm{CO}_{2}$. Chronotropic responses were assessed by extracellular recordings for 10 minutes before and after the culture medium was replaced by a medium containing $10^{-6} \mathrm{M}$ isoproterenol, $10^{-6} \mathrm{M}$ carbamylcholine, $10^{-5} \mathrm{M}$ 3-isobutyl-1-methylxanthine (IBMX), or $10^{-6} \mathrm{M}$ forskolin (all from Sigma Chemical Co.).

Intracellular calcium $\left(\left[\mathrm{Ca}^{2+}\right]_{i}\right)$ transients. The EBs were loaded with fura 2-AM (Molecular Probes Inc., Eugene, Oregon, USA) for 25 minutes at room temperature $\left(24-25^{\circ} \mathrm{C}\right)$ at a final concentration of $5 \mu \mathrm{M}$, in a $1: 1$ mixture of Tyrode's solution. EBs were then transferred to a nonfluorescent chamber mounted on the stage of an inverted microscope (Diaphot 300; Nikon Inc., Tokyo, Japan). The chamber was perfused with Tyrode's solution at a rate of $1 \mathrm{ml} / \mathrm{min}$. Experiments were performed at $37^{\circ} \mathrm{C}$. Fura 2 fluorescence was measured using a dual-wavelength system (Deltascan; Photon Technology International, Lawrenceville, New Jer-

Table 1

PCR primers used in this study

\begin{tabular}{|c|c|c|c|}
\hline Gene product & Primer & reaction condition & Product size (bp) \\
\hline hANP & $\begin{array}{l}\text { GAACCAGAGGGGAGAGACAGAG } \\
\text { CCCTCAGCTTGCTIITTAGGAG }\end{array}$ & 35 cycles at $61^{\circ} \mathrm{C}$ in $1 \mathrm{mM} \mathrm{MgCl}_{2}$ & 406 \\
\hline MLC-2A & $\begin{array}{l}\text { ACAGAGTTTATTGAGGTGCCCC } \\
\text { AAGGTGAAGTGTCCCAGAGG }\end{array}$ & 35 cycles at $61^{\circ} \mathrm{C}$ in $1 \mathrm{mM} \mathrm{MgCl}_{2}$ & 381 \\
\hline MLC-2V & $\begin{array}{l}\text { TATTGGAACATGGCCTCTGGAT } \\
\text { GGTGCTGAAGGCTGATTACGTT }\end{array}$ & 35 cycles at $61^{\circ} \mathrm{C}$ in $1 \mathrm{mM} \mathrm{MgCl}_{2}$ & 382 \\
\hline GATA4 & $\begin{array}{l}\text { AGACATCGCACTGACTGAGAAC } \\
\text { GACGGGTCACTATCTGTGCAAC }\end{array}$ & 30 cycles at $60^{\circ} \mathrm{C}$ in $1 \mathrm{mM} \mathrm{MgCl}_{2}$ & 475 \\
\hline$\alpha$-Myosin heavy chain & $\begin{array}{l}\text { GTCATTGCTGAAACCGAGAATG } \\
\text { GCAAAGTACTGGATGACACGCT }\end{array}$ & 40 cycles at $61^{\circ} \mathrm{C}$ in $2 \mathrm{mM} \mathrm{MgCl}_{2}$ & 413 \\
\hline Oct-4 & $\begin{array}{l}\text { GAGAACAATGAGAACCTTCAGGAGA } \\
\text { TTCTGGCGCCGGTTACAGAACCA }\end{array}$ & 35 cycles at $55^{\circ} \mathrm{C}$ in $1.5 \mathrm{mM} \mathrm{MgCl}_{2}$ & 219 \\
\hline $\mathrm{Nkx2.5}$ & $\begin{array}{l}\text { CTTCAAGCCAGAGGCCTACG } \\
\text { CCGCCTCTGTCTTCTTCAGC }\end{array}$ & 35 cycles at $55^{\circ} \mathrm{C}$ in $1.5 \mathrm{mM} \mathrm{MgCl}_{2}$ & 233 \\
\hline$c T n T$ & $\begin{array}{l}\text { GGCAGCGGAAGAGGATGCTGAA } \\
\text { GAGGCACCAAGTTGGGCATGAACGAC }\end{array}$ & 35 cycles at $60^{\circ} \mathrm{C}$ in $1.5 \mathrm{mM} \mathrm{MgCl}_{2}$ & 150 \\
\hline cTnl & $\begin{array}{l}\text { CCCTGCACCAGCCCCAATCAGA } \\
\text { CGAAGCCCAGCCCGGTCAACTС }\end{array}$ & 35 cycles at $60^{\circ} \mathrm{C}$ in $1.5 \mathrm{mM} \mathrm{MgCl}_{2}$ & 250 \\
\hline GAPDH & $\begin{array}{l}\text { AGCCACATCGCTCAGACACC } \\
\text { GTACTCAGCGGCCAGCATCG }\end{array}$ & 25 cycles at $61^{\circ} \mathrm{C}$ in $1.5 \mathrm{mM} \mathrm{MgCl}_{2}$ & 302 \\
\hline
\end{tabular}

hANP, human ANP; Oct-4, octamer-binding protein 4. ${ }^{A}$ Ref. $15 .{ }^{B}$ Ref. 16. ${ }^{\text {CRef. }} 17 .{ }^{D}$ Ref. 12. 


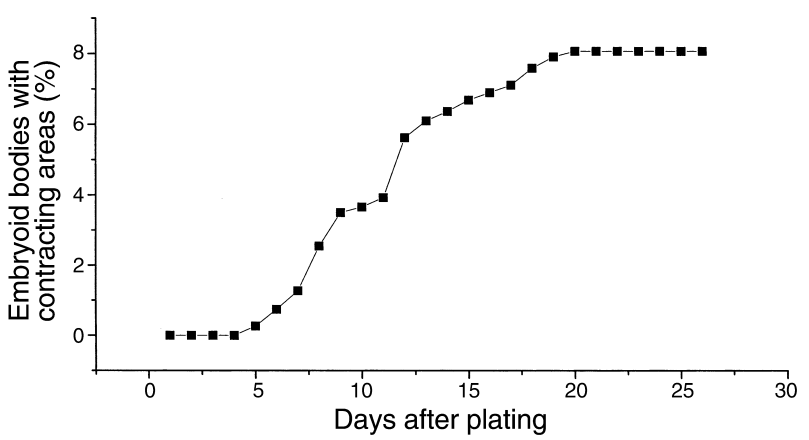

Figure 2

Cumulative percentage of EBs containing spontaneously contracting areas as a function of the number of days after plating of the $E B$.

sey, USA) as described previously (19). Briefly, two different wavelengths (340 and $380 \mathrm{~nm}$ ) were used for excitation, and the emitted fluorescence $(510 \mathrm{~nm})$ was collected and detected by a photomultiplier tube $(710$ PMT, photomultiplier detection system; Photon Technology International). Raw data were stored for off-line analysis by the FeliX software (Photon Technology International) as 340 and $380 \mathrm{~nm}$ counts and as the ratio $R=F_{340} / F_{380}$. The Savitzky-Golay smoothing algorithm was used to reduce noise level.

Statistical analysis. Data are expressed as mean \pm SD. To assess possible chronotropic effects, the average spontaneous beating rate was compared before and after drug application using two-tailed paired Student's $t$ test. $P$ values less than 0.05 were considered significant.

\section{Results}

Human ES cell-derived cardiomyocytes form spontaneously contracting areas. Figure $1 \mathrm{~b}$ depicts typical examples of ES colonies grown on top of the MEF feeder layer, the formation of EBs during the suspension phase, and an EB containing a contracting area after plating. Rhythmically contracting areas appeared at 4-22 days after plating. Figure 2 illustrates the cumulative percentage of EBs containing contracting areas as a function of the time after plating. Such contracting areas appeared in $153(8.1 \%)$ of the 1,884 EBs studied. At $11-12$ days after plating, $50 \%$ of the plateau value was reached. The contracting areas usually appeared in the outgrowth of the EB, with a diameter range of $0.2-2 \mathrm{~mm}$, and continued to beat vigorously for up to 5 weeks (the longest period studied). DMSO at a concentration of $0.75 \%$ did not cause a major change in the percentage of beating EBs, with 46 of 454 (10.1\%) of the EBs demonstrating contracting areas.

Structural analysis. Light microscopy revealed that the contracting areas were composed mainly of relatively small mononuclear cells, $10-30 \mu \mathrm{m}$ in diameter, with round or rod-shaped morphology. Transmission electron microscopy of these cells revealed mononuclear cells, with parallel arrays of myofibrillar bundles oriented in an irregular manner in some cells (Figure 3a), whereas more mature sarcomeric organization was apparent in others (Figure 3, b and c). The degree of myofibrillar organization varied within different areas of the same cell, among different cells in the same EB, and among different EBs. Nevertheless, in general, a shift from an immature phenotype manifested by disorganized myofibrillar stacks in early stage EBs (Figure 3a) to a more organized sarcomeric structure in later stage EBs (Figure 3, b and c) was noted. In some foci, the formation of early and more developed $\mathrm{Z}$ bands could be observed (Figure 3, b and c). The intercalated disc, another cellular structure that characteristically appears during in vivo cardiomyocyte differentiation, was observed in many of the differentiating

\section{Figure 3}

Ultrastructural analysis of ES-derived cardiomyocytes. (a) Transmission electron micrograph of sectioned beating EB 10 days after plating. Relatively unorganized myofibrillar bundles can be seen in some myocytes. (b) A cell 27 days after plating, displaying a more mature sarcomeric organization. (c) A different cell from the same EB as in $\mathbf{b}$, demonstrating more organized sarcomeres and Z-bands (arrow). (d) High-power electron micrograph showing the presence of a gap junction (arrow) from a cell 16 days after plating. (e) High-power electron micrograph showing the presence of desmosomes (arrow) from a cell 16 days after plating.
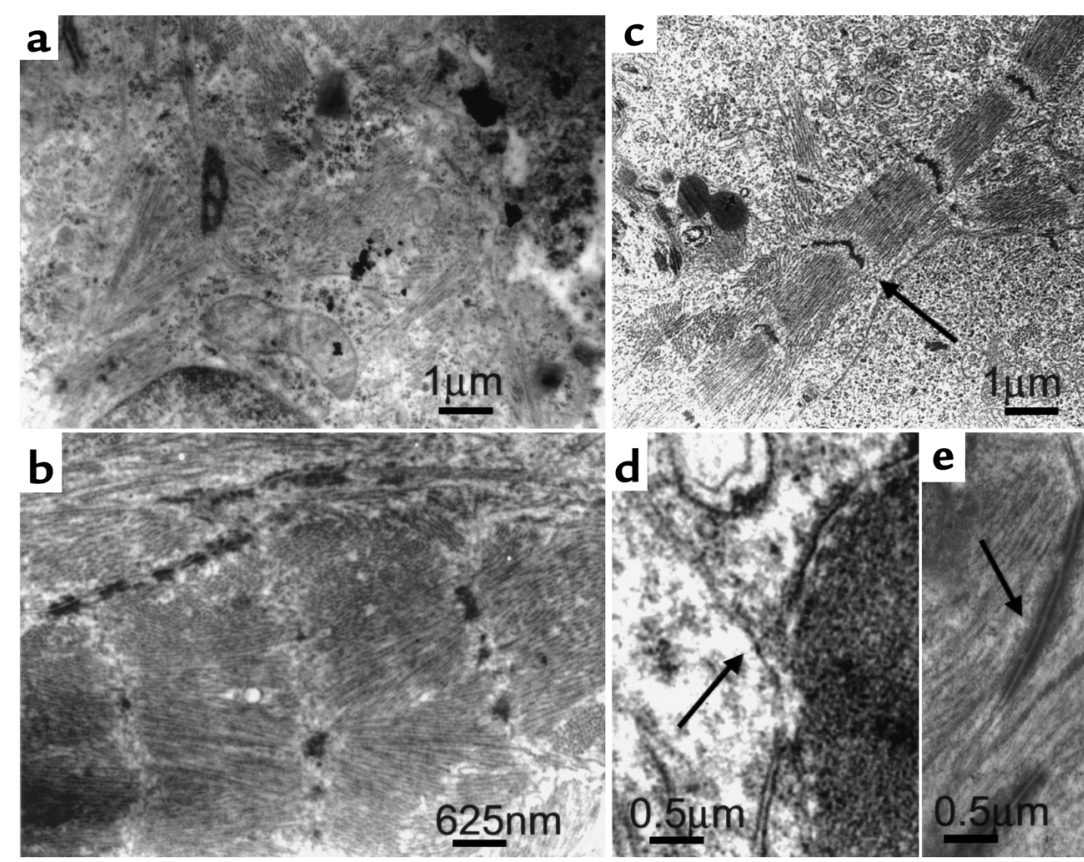

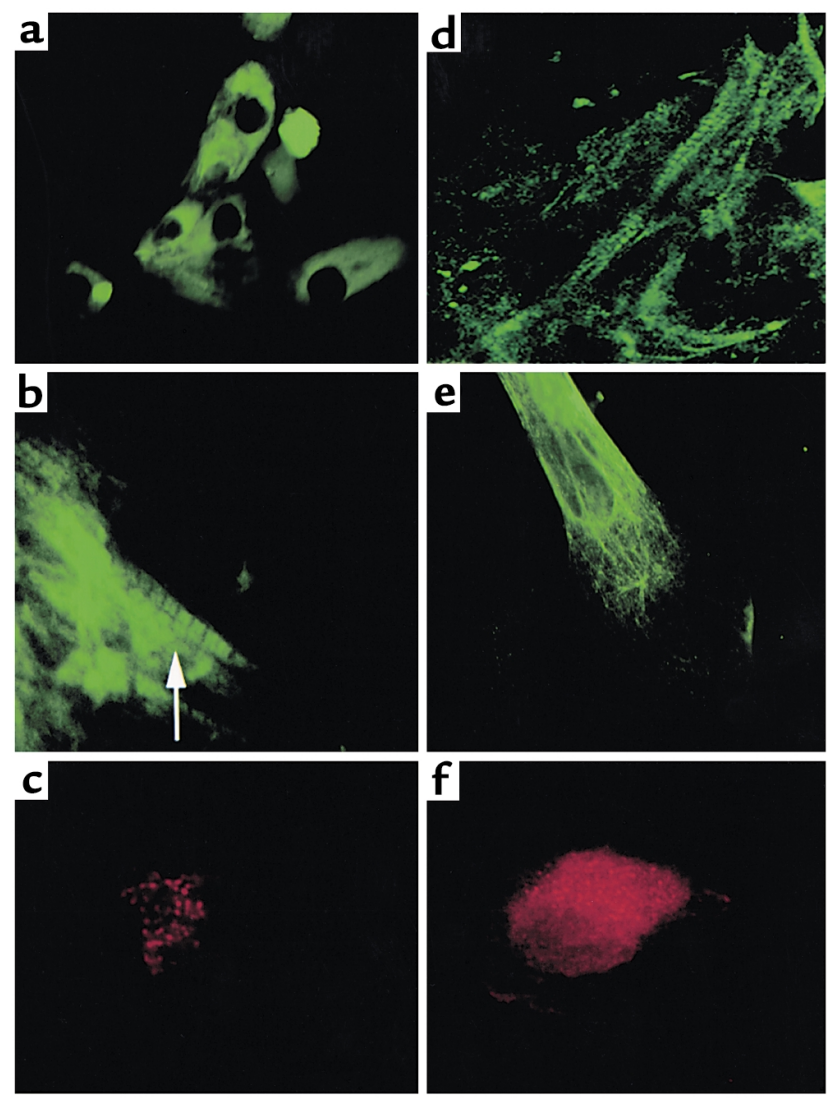

EBs. Hence, intercalated discs composed of gap junctions and desmosomes were observed to connect adjacent cells (Figure 3, d and e).

Using immunocytochemistry, the presence of cardiacspecific proteins and their spatial organization were studied in dispersed cells forming contracting EBs. Figure $4 \mathrm{a}$ shows positive immunostaining of dispersed myocytes with anti-cardiac $\alpha / \beta$-myosin heavy chain mAb's. Varying degrees of myofibrillar organization were noted among the cells. The staining patterns ranged from cytoplasmic clumps in some cells to nonparallel bundles of elongated fibrillar structures in others. Some of these bundles displayed an early striated pattern (Figure 4b). The contracting myocytes also stained positively with anti- $\alpha$-actinin mAb's (Figure $4 c$ ), anti-cTnI mAb's (Figure 4d), and anti-desmin mAb's (Figure 4e), with different cells demonstrating varying degrees of sarcomeric organization. In addition, the positive staining by anti-ANP (Figure 4f) suggested the presence of cytoplasmic ANP granules. In contrast to the positive staining with cardiac-specific proteins, cells from the contracting areas did not demonstrate nebulin immunoreactivity, confirming the cardiomyocytic, rather than skeletal, nature of the cells.

To determine the percentage of cardiomyocytes in the contracting areas, the regions exhibiting spontaneous contracting activity were microdissected, enzymatically dispersed, and plated at low density to allow identification of individual cells by immunocyto-

\section{Figure 4}

Immunostaining of ES cell-derived cardiomyocytes. (a) Immunostaining of dispersed cells from a beating EB (day 16 after plating) with anti-cardiac $\alpha / \beta$-myosin heavy chain mAb's. Several cells stained positively. $\times 40$. (b) Higher magnification of a cardiomyocyte in a more developed stage (day 16 after plating). Note the appearance of early striation pattern (arrow). $\times 63$. (c) Positive staining with anti-sarcomeric $\alpha$-actinin mAb's (day 17 after plating). $\times 63$. (d) Positive staining with cTnl mAb's (day 30 after plating). $\times 63$. (e) Positive staining with anti-desmin mAb's (day 18 after plating). $\times 63$. (f) Positive staining with anti-ANP antibodies (day 16 after plating). $\times 63$.

chemistry. Using cTnI mAb's, the percentage of positively stained cells was $29.4 \%$.

Gene expression studies. The expression of several cardiac-specific genes was assessed in the human ES cell-derived cardiomyocytes and in undifferentiated ES cells using RT-PCR. As shown in Figure 5, myocytes from contracting EBs expressed the cardiac transcription factors GATA4 and $\mathrm{Nkx} 2.5$ as well as the cardiac-specific genes $\mathrm{CTnI}$, cardiac troponin $\mathrm{T}$ (cTnT), atrial myosin light chain (MLC-2A), ventricular myosin light chain (MLC-2V), and $\alpha$-myosin heavy chain. MLC-2A expression was also noted in the undifferentiated ES cells, but was markedly increased in the contracting EBs. Octamer-binding protein 4, a marker of undifferentiated cells, was expressed in the ES cells. This expression significantly declined in the contracting EBs. The housekeeping gene GAPDH served as an internal control.

Measurements of $\left[\mathrm{Ca}^{2+}\right]_{i}$ transients. $\left[\mathrm{Ca}^{2+}\right]_{i}$ transients were measured from spontaneously contracting EBs using fura-2AM $(n=7)$. The average spontaneous beating rate was $0.9 \pm 0.11 \mathrm{~Hz}$. Figure 6a displays typical recordings with an initial rise in systolic $\left[\mathrm{Ca}^{2+}\right]_{\mathrm{i}}$ and a slower decay. The time to peak systolic $\left[\mathrm{Ca}^{2+}\right]_{i}$

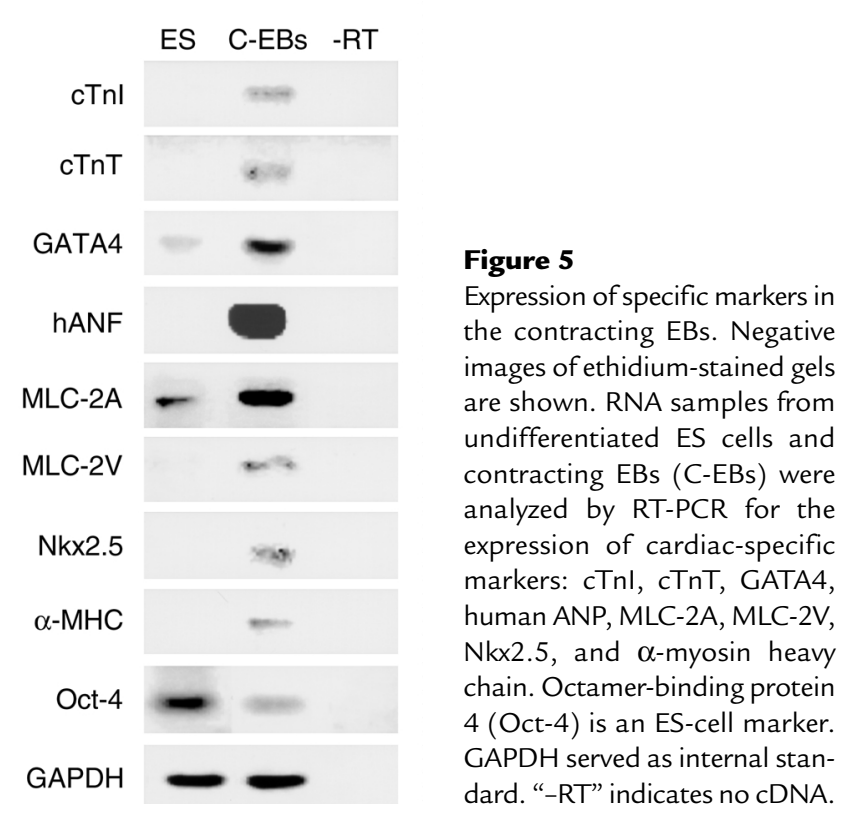



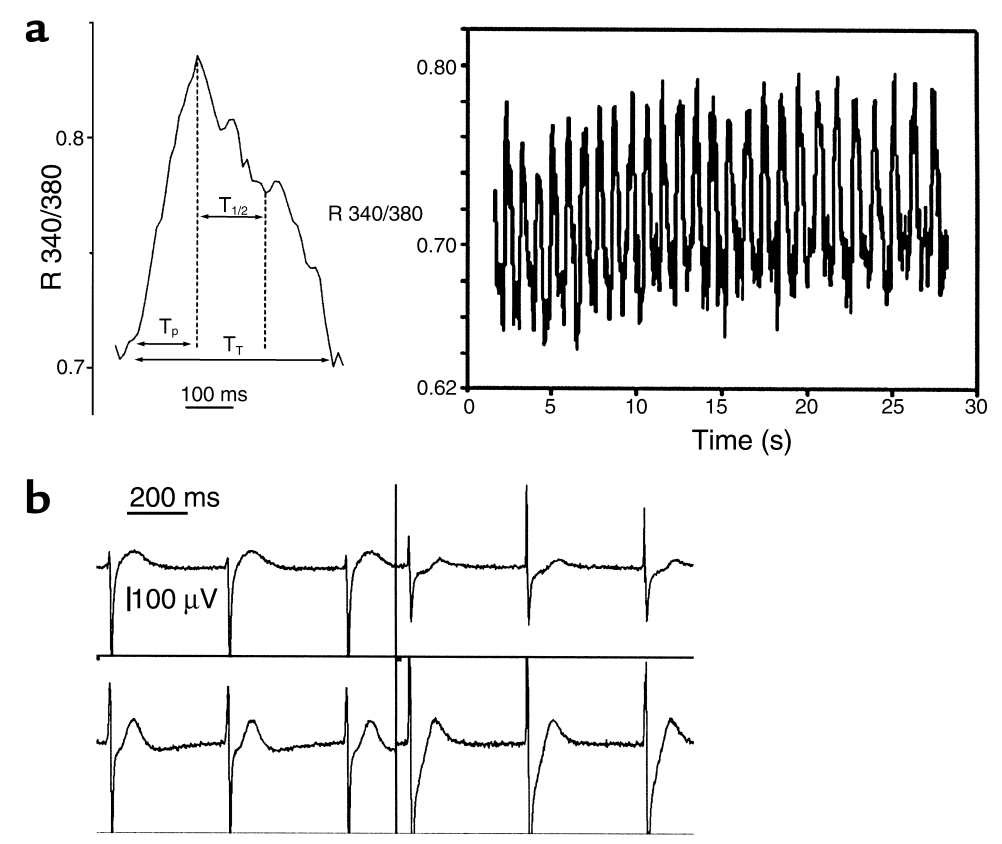

\section{Figure 6}

Functional analysis of contracting area within the EBs. (a) Calcium transients of human ES cell-derived cardiomyocytes as determined by fura-2 fluorescence. A typical calcium transient is shown on the left, and continuous recording is shown on the right. $T_{p}$, time to peak; $T_{t}$, total transient time; $T_{1 / 2}$, time to half-peak relaxation. (b) Typical extracellular electrophysiological recordings from different areas of the EB. Note the presence of a sharp and slow component. R, ratio. reversed by application of the muscarinic antagonist atropine. All of these responses were prompt, occurring within 90 seconds of drug application.

\section{Discussion}

ES cells provide a unique cellular system to study commitment and differentiation of embryonic tissue under in vitro conditions. Cultured as EBs, murine ES cells recapitulate the development of cardiomyocytes from very early cardiac precursor cells to terminally differentiated cells (7). The advent of the murine ES model has thus provided important insights into the early steps of cardiomyogenesis, including the origin, commitment, and differentiation of cardiac cells, patterns of gene expression, myofibrillogenesis, ion channel development and function, and calcium handling (4-9). Nevertheless, there are significant differences between human and murine development, and, for ethical reasons, it is essentially impossible to study the postimplantation human embryo directly.

In this report, we have described a reproducible novel system in which human ES cells differentiate into cardiomyocytic tissue. Our results demonstrate that the spontaneously contract-

averaged $130 \pm 27$ milliseconds, the time to half-peak relaxation was $143 \pm 94$ milliseconds, and total transient length was $465 \pm 180$ milliseconds. In all cases, the $\left[\mathrm{Ca}^{2+}\right]_{i}$ signals were synchronous with the contraction rate observed microscopically.

Extracellular electrophysiological recordings and chronotropic studies. Extracellular recordings from the contracting areas displayed electrograms consisting of a sharp component with a peak-to-peak amplitude of $630 \pm 33 \mu \mathrm{V}$ lasting $30 \pm 25$ milliseconds, followed by a slow component of $347 \pm 120$ milliseconds, representing the depolarization and repolarization processes, respectively (Figure 6b). The average spontaneous beating rate was $94 \pm 33$ beats per minute $(n=8)$ and was stable (mean frequency SD of 1.55 beats per minute) during a recording period of 20 minutes in all EBs studied.

Positive and negative chronotropic responses were observed after administration of the $\beta$-agonist isoproterenol and the muscarinic agonist carbamylcholine, respectively. Isoproterenol at $10^{-6} \mathrm{M}$ significantly increased spontaneous contraction rate to $146 \% \pm 43 \%$ of its baseline value $(n=8 ; P<0.01)$. Similarly, the direct adenylate cyclase activator forskolin and the phosphodiesterase inhibitor IBMX increased spontaneous contraction rate to $182 \% \pm 48 \%(n=6$; $P<0.01)$ and $152 \% \pm 77 \%(n=6 ; P<0.05)$ of its initial value. In contrast, the muscarinic agonist carbamylcholine at $10^{-6} \mathrm{M}$ decreased the rate to $78 \% \pm 20 \%$ of its initial value $(n=6 ; P<0.05)$. The latter effect was ing tissue within the developing EBs contain myocytes portraying structural and functional properties consistent with early-stage cardiac tissue.

Several lines of evidence confirm the cardiomyocytic nature of these cells. Ultrastructural analysis of the differentiating cardiomyocytes showed that these cells were mainly mononuclear and round or rod-shaped, contained different degrees of myofibrillar bundle organization, and exhibited nascent intercalated discs. These myofibrillar structures stained positively with anti-human cardiac myosin heavy chain, anti- $\alpha$ actinin, anti-desmin, and anti-cTnI mAb's. The cells, however, did not exhibit immunoreactivity with antinebulin mAb's, a specific skeletal muscle sarcomeric protein shown to be expressed early in skeletal myoblast differentiation (20).

These results are consistent with ultrastructural properties of early-stage cardiomyocytes and with the developmental process of myofibrillar assembly. Previous studies demonstrated that during in vivo cardiomyogenesis, myofibrils are initially distributed in sparse, irregular myofibrillar arrays, which gradually mature into parallel arrays of myofibrils and ultimately align into densely packed sarcomeres $(21,22)$.

ES-derived cardiomyocytes express a number of cardiomyocyte-specific genes, including the transcription factors GATA4 and Nkx2.5, which play a significant role in heart formation in vertebrates (23). In addition, in this study the cardiac proteins ANP, cTnI, cTnT, 
MLC-2A, MLC-2V, and $\alpha$-myosin heavy chain were also found to be expressed. MLC-2A expression was also noted in the undifferentiated ES cells, but was markedly increased in the contracting EBs. This expression probably reflects some background differentiation of the ES cells on the feeder layer. The presence of both MLC-2A and MLC-2V might suggest the presence of a number of cardiomyocytic cell types within the contracting areas.

The extracellular recordings, the $\left[\mathrm{Ca}^{2+}\right]_{\mathrm{i}}$ transients, and the pharmacological studies clearly demonstrate that the contracting areas within the EBs displayed physiological properties consistent with cardiomyocytic tissue and significantly differed from noncardiac (skeletal or smooth) muscle. All components of normal cardiac excitation-contraction coupling were demonstrated within this tissue, namely electrical activation, increase in $\left[\mathrm{Ca}^{2+}\right]_{\mathrm{i}}$ and the resulting contraction.

Little is known about calcium handling in the normal developing human heart. $\left[\mathrm{Ca}^{2+}\right]_{i}$ transients in adult human atrial tissue, studied using fura-2 (24), were similar to the ones recorded in the present study with respect to total duration of the $\left[\mathrm{Ca}^{2+}\right]_{\mathrm{i}}$ transient and to time of half-peak calcium relaxation. However, the time to peak transient in the EBs $(130 \pm 27$ milliseconds) was longer than the one obtained in human atrial myocytes (52.5 \pm 3.1 milliseconds). This difference may have been the result of a number of causes. In the murine ES model, the contracting areas within EBs were shown to be a mixture of atrial, ventricular, and sinus nodal cells (25). Given that different cardiomyocyte types display different calcium characteristics (26), it is possible that the $\left[\mathrm{Ca}^{2+}\right]_{\mathrm{i}}$ transients measured in whole beating EBs, in the current study, represent a superposition of a mixed population of myocytes with fast and slow cell characteristics. Alternatively, the lower rate in $\left[\mathrm{Ca}^{2+}\right]_{i}$ rise may have been due to lower efficiency and immaturity of the calcium machinery in early developing cardiac cells.

The extracellular recordings demonstrated a sharp and a slow component most probably consistent with a relatively long action potential duration characteristic of cardiomyocytes. Fetal and neonatal human skeletal muscle cells in culture, on the other hand, display a much shorter action potential, with mean action potential duration at $50 \%$ of depolarization $\left(\mathrm{APD}_{50}\right)$ of only 7.5 milliseconds (27). The positive and negative chronotropic responses to isoproterenol and carbamylcholine demonstrated the presence of functional adrenergic and cholinergic receptors, respectively, in pacemaker cells. A major pathway of the $\beta$-adrenoreceptor-dependent chronotropic response is the activation of adenylate cyclase and the consequent rise in cytosolic cAMP and stimulation of protein kinase. The positive chronotropic effect exerted by forskolin, a direct activator of adenylate cyclase, and by IBMX, a phosphodiesterase inhibitor, suggests that this signaling pathway is already present early in human cardiomyocytic differentiation.
Differences between the murine and human models. Several differences distinguish human and mouse ES cells. Human ES cells have a slightly different morphology and form flatter colonies. The stage-specific embryonic antigen-3 and antigen-4, as well as TRA-1-60 and TRA-1-81, have been shown to be expressed by human but not by mouse ES cells (10). In contrast to the mouse, human ES cells differentiate when cultured in the absence of MEF feeder layer, even in the presence of leukemia inhibitory factor.

The differences between the human and mouse cell lines are not limited to the stem cells. In the normal embryo, heart formation begins with the initiation of differentiation by myocardial and endocardial precursors and leads up to the formation of the cardiac valves. These events cover the first 12 days in the life of a mouse embryo and the first 35 days in the life of a human embryo (23). It is not surprising, therefore, that differentiation of human ES cells into cardiomyocytes proceeds at a slower rate than in mouse ES cells. In the mouse model, ES cells are cultivated in hanging drops for 2 days and are further cultivated as EBs in suspension for 5 days. Spontaneously contracting areas appear 1 day after plating, and, within $2-10$ days, $80-90 \%$ of EBs reveal pulsating areas (28). In the human ES cell differentiation system described here, cells were grown in suspension for 10 days, and spontaneous contractions did not commence before day 4 after plating, with the median value being 11 days. Furthermore, only $8.1 \%$ of EBs revealed pulsating areas. These variations may represent differences between the species, differences between the cell lines, or some yet undetermined factor required in the in vitro differentiation of human ES cells. Several factors that may be optimized in the future to increase cardiomyocytic yield include different serum content, length of suspension period, the use of growth factors, or the use of supporting stroma.

Morphologically, in vitro differentiation of human and mouse ES cells appears to follow parallel pathways. The assembly of the Z-line from periodically aligned $Z$-bodies and the transition from disorganized myofibrils to the more organized sarcomeric pattern described here have also been noted in the mouse model (7). In the two models, different degrees of myofibrillar assembly coexist within adjacent cardiomyocytes in the same EB and within the same cell. Nevertheless, in the human model, ultrastructural maturation proceeded much more slowly, seemed more heterogeneous, and did not reach the fully mature adult phenotype during the observation period.

Possible research and clinical applications. Establishment of a cardiomyocyte differentiating system from human ES cells may become a powerful tool for understanding cardiac development and function, as human cardiomyocytes are not accessible in adequate quantities for research, especially at this early stage of differentiation. Specifically, important insights may be gathered with regard to the mechanisms involved in cardiac lineage commitment, the process of sarcomeric organization, 
action potential and ion channel development, pacemaker organization, calcium handling, and establishment of the adrenergic and cholinergic receptors, as well as the molecular signals involved in these processes.

Another attractive application of these cells is in cell replacement therapy. Adult cardiomyocytes withdraw permanently from the cell cycle during differentiation; hence, any significant loss of cardiomyocytes (as occurs, for example, during myocardial infarction) is irreversible and leads to diminished cardiac function and to the development of progressive heart failure. A potential novel approach for this situation may be the implantation of myogenic cells within the infarcted tissue $(1,2$, $29,30)$. Although a number of myocyte preparations have been suggested, the inherent electrophysiological, structural, and contractile properties of cardiomyocytes strongly suggest that they may be the ideal donor cell type. Because human fetal cardiomyocytes cannot be obtained in sufficient numbers for clinical purposes, the use of cardiomyocytes derived from ES cell lines may become an attractive option. Nevertheless, several obstacles must be overcome in order to achieve this goal, including the generation of enriched and relatively pure cardiomyocyte cultures and the establishment of different strategies to counter immune rejection.

In summary, this study described the generation of a reproducible cardiomyocyte differentiation system from human ES cells. The generated myocytes were shown to display functional and structural properties consistent with early-stage cardiomyocytes. The establishment of this unique system may have an important impact on the understanding of human cardiac development and function, and may provide a powerful research and clinical tool in several fields such as pharmacological and toxicological testing, functional genomics, early cardiomyogenesis, cell therapy, and tissue engineering.

\section{Acknowledgments}

This research was supported in part by the Israel Science Foundation; by the Technion V.P.R. Fund-Malat Family, Loewengart, and Hirshenstrauss-Gutman Medical Research Fund (L. Gepstein); and by the fund for Medical Research and Development of Infrastructure and Health Services, Rambam Medical Center (J. ItskovitzEldor). We thank Pesisa Shentzere for technical support, and Ruth Singer for editing of the manuscript.

1. Soonpaa, M.H., Koh, G.Y., Klug, M.G., and Field, L.J. 1994. Formation of nascent intercalated disks between grafted fetal cardiomyocytes and host myocardium. Science. 264:98-101.

2. Leor, J., Patterson, M., Quinones, M.J., Kedes, L.H., and Kloner, R.A. 1996. Transplantation of fetal myocardial tissue into the infarcted myocardium of rat. A potential method for repair of infarcted myocardium? Circulation. 94(Suppl. 9):II332-II336.

3. Evans, M.J., and Kaufman, M.H. 1981. Establishment in culture of pluripotential cells from mouse embryos. Nature. 292:154-156.

4. Sánchez, A., Jones, W.K., Gulick, J., Doetschman, T., and Robbins, J. 1991. Myosin heavy chain gene expression in mouse embryoid bodies. An in vitro developmental study. J. Biol. Chem. 266:22419-22426.

5. Metzger, J.M., Lin, W.-I., Johnston, R.A., Westfall, M.V., and Samuelson, L.C. 1995. Myosin heavy chain expression in contracting myocytes isolated during embryonic stem cell cardiogenesis. Circ. Res. 76:710-719.

6. Maltsev, V.A., Wobus, A.M., Rohwedel, J., Bader, M., and Hescheler, J. 1994. Cardiomyocytes differentiated in vitro from embryonic stem cells developmentally express cardiac-specific genes and ionic currents. Circ. Res. 75:233-244.

7. Hescheler, J., et al. 1997. Embryonic stem cells: a model to study structural and functional properties in cardiomyogenesis. Cardiovasc. Res. 36:149-162.

8. Maltsev, V.A., Ji, G.J., Wobus, A.M., Fleischmann, B.K., and Hescheler, J. 1999. Establishment of $\beta$-adrenergic modulation of L-type $\mathrm{Ca}^{2+}$ current in the early stages of cardiomyocyte development. Circ. Res. 84:136-145.

9. Viatchenko-Karpinski, S., et al. 1999. Intracellular $\mathrm{Ca}^{2+}$ oscillations drive spontaneous contractions in cardiomyocytes during early development. Proc. Natl. Acad. Sci. USA. 96:8259-8264.

10. Thomson, J.A., et al. 1998. Embryonic stem cell lines derived from human blastocysts. Science. 282:1145-1147.

11. Reubinoff, B.E., Pera, M.F., Fong, C.-Y., Trounson, A., and Bongso, A. 2000. Embryonic stem cell lines from human blastocysts: somatic differentiation in vitro. Nat. Biotech. 18:399-404.

12. Itskovitz-Eldor, J., et al. 2000. Differentiation of human embryonic stem cells into embryoid bodies compromising the three embryonic germ layers. Mol. Med. 6:88-95.

13. Schuldiner, M., Yanuka, O., Itskovitz-Eldor, J., Melton, D.A., and Benvenisty, N. 2000. Effects of eight growth factors on the differentiation of cells derived from human embryonic stem cells. Proc. Natl. Acad. Sci. USA. 97:11307-11312.

14. Amit, M., et al. 2000. Clonally derived human embryonic stem cell lines maintain pluripotency and proliferative potential for prolonged periods of culture. Dev. Biol. 227:271-278.

15. Abdel-Rahman, B., Fiddler, M., Rappolee, D., and Pergament, E. 1995. Expression of transcription regulating genes in human preimplantation embryos. Hum. Reprod. 10:2787-2792.

16. Shiojima, I., et al. 1996. Molecular cloning and characterization of human cardiac homeobox gene CSX1. Circ. Res. 79:920-929.

17. Ricchiuti, V., Apple, F.S. 1999. RNA expression of cardiac troponin T isoforms in diseased human skeletal muscle. Clin. Chem. 45:2129-2135.

18. Igelmund, P., et al. 1999. Action potential propagation failures in longterm recordings from embryonic stem cell-derived cardiomyocytes in tissue culture. Pflugers Arch. 437:669-679.

19. Felzen, B., et al. 1998. Fas (CD95/Apo-1)-mediated damage to ventricular myocytes induced by cytotoxic $\mathrm{T}$ lymphocytes from perforin-deficient mice. A major role for inositol 1,4,5-triphosphate. Circ. Res. 82:438-450.

20. Begum, S., et al. 1998. Differentiation of muscle-specific proteins in chicken somites as studied by immunofluorescence microscopy. Cell Tissue Res. 293:305-311.

21. Manasek, F.J. 1970. Histogenesis of the embryonic myocardium. Am. J. Cardiol. 25:149-168.

22. Chacko, K.J. 1976. Observations on the ultrastructure of developing myocardium of rat embryos. J. Morphol. 150:681-709.

23. Stainier, D.Y. 2001. Zebrafish genetics and vertebrate heart formation. Nat. Rev. Genet. 2:39-48.

24. Brixius, K., Pietsch, M., Hoischen, S., Müller-Ehmsen, J., and Schwinger, R.H.G. 1997. Effect of inotropic interventions on contraction and $\mathrm{Ca}^{2+}$ transients in the human heart. J. Appl. Physiol. 83:652-660.

25. Maltsev, V.A., Rohwedel, J., Hescheler, J., and Wobus, A.M. 1993. Embryonic stem cells differentiate in vitro into cardiomyocytes representing sinusnodal, atrial and ventricular cell types. Mech. Dev. 44:41-50.

26. Kolossov, E., et al. 1998. Functional characteristics of ES cell-derived cardiac precursor cells identified by tissue-specific expression of the green fluorescent protein. J. Cell Biol. 143:2045-2056.

27. Iannaccone, S.T., Li, K., and Sperelakis, N. 1987. Transmembrane electrical characteristics of cultured human skeletal muscle cells. J. Cell. Physiol. 133:409-413.

28. Wobus, A.M., Wallukat, G., and Hescheler, J. 1991. Pluripotent mouse embryonic stem cells are able to differentiate into cardiomyocytes expressing chronotropic responses to adrenergic and cholinergic agents and $\mathrm{Ca}^{2+}$ channel blockers. Differentiation. 48:173-182.

29. Taylor, D.A., et al. 1998. Regenerating functional myocardium: improved performance after skeletal myoblast transplantation. Nat. Med. 4:929-933.

30. Klug, M.G., Soonpaa, M.H., Koh, G.Y., and Field, L.J. 1996. Genetically selected cardiomyocytes from differentiating embryonic stem cells form stable intracardiac grafts. J. Clin. Invest. 98:216-224. 